\title{
REDUCING MOD $p$ COMPLEX REPRESENTATIONS OF FINITE REDUCTIVE GROUPS
}

\author{
G. LUSZTIG \\ Dedicated to the memory of Jim Humphreys
}

\begin{abstract}
We state a conjecture on the reduction modulo the defining characteristic of a unipotent representation of a finite reductive group.
\end{abstract}

\section{INTRODUCTION}

0.1 . Let $\mathbf{k}$ be an algebraic closure of the finite field with $p$ elements ( $p$ is a prime number). Let $\mu$ be the group of roots of 1 in $\mathbf{C}$. We fix a surjective homomorphism $\phi: \mu \rightarrow \mathbf{k}^{*}$ whose kernel is the set of roots of 1 of order a power of $p$. Let $\Gamma$ be a finite group. Let $\mathcal{R} \Gamma$ (resp. $\mathcal{R}_{p} \Gamma$ ) be the Grothendieck group of virtual (finite dimensional) representations of $\Gamma$ over $\mathbf{C}($ resp. over $\mathbf{k})$ and let $\mathcal{R}^{+} \Gamma\left(\operatorname{resp} . \mathcal{R}_{p}^{+} \Gamma\right)$ be the subset of $\mathcal{R} \Gamma$ (resp. $\mathcal{R}_{p} \Gamma$ ) given by actual representations of $\Gamma$ over $\mathbf{C}$ (resp. over $\mathbf{k}$ ). Following Brauer and Nesbitt $[\mathrm{BN}]$ there is a well defined map $\rho \mapsto \underline{\rho}$ from $\mathcal{R}^{+} \Gamma$ to $\mathcal{R}_{p}^{+} \Gamma$ characterized by the following property: for any $g \in \Gamma$ the eigenvalues of $g$ on $\rho$ are obtained by applying $\phi$ to the eigenvalues of $g$ on $\rho$. We say that $\rho$ is the reduction modulo $p$ of $\rho$. In the remainder of this paper we assume that $\bar{\Gamma}=G\left(F_{p}\right)$ is the group of $F_{p}$-rational points of an almost simple simply connected linear algebraic group $G$ over $\mathbf{k}$ with a given split $F_{p}$-structure with $p$ sufficiently large. Our goal is to present some remarks on the map $\rho \mapsto \underline{\rho}$ in this case.

0.2. Assume that $G=S L_{2}(\mathbf{k})$. Assume that $\rho \in \mathcal{R}^{+} \Gamma$ is irreducible. If $\rho$ has dimension $1, p,(p+1) / 2$ or $(p-1) / 2$, then $\rho$ is irreducible. If $\operatorname{dim} \rho=p+1$, then $\rho$ has two composition factors, of dimension $c, p+1-c$ with $2 \leq c \leq(p-1) / 2$ (and any such $c$ occurs). If $\operatorname{dim} \rho=p-1$, then either $\rho$ has two composition factors, of dimension $c, p-1-c$ with $2 \leq c \leq(p-3) / 2$ (and any such $c$ occurs) or $\rho$ is irreducible. These results can be found in the paper [BN] of Brauer and Nesbitt (they actually consider the group $P S L_{2}\left(F_{p}\right)$ instead of $S L_{2}\left(F_{p}\right)$ but their method applies also to $\left.S L_{2}\left(F_{p}\right)\right)$.

0.3. Assume that $G=S L_{3}(\mathbf{k})$. In the case where $\rho$ is an irreducible representation in $\mathcal{R}^{+} \Gamma$ which has a line stable under the upper triangular subgroup, the complete description of the composition factors of $\rho$ was given in [CL] (written in 1973). For one of the cuspidal irreducible representations $\rho$ of $\Gamma, \underline{\rho}$ has exactly two composition factors (except when $p=2$ when $\rho$ is irreducible), as stated in [L1] (where the case $p=2$ was overlooked); one has dimension $p(p-1)(2 p-1) / 2$ and the other (when $p>2)$ has dimension $(p-1)(p-2) / 2$. This is analogous to the cuspidal irreducible

Received by the editors July 31, 2020, and, in revised form, December 4, 2020.

2020 Mathematics Subject Classification. Primary 20 G99.

The author was supported by NSF grant DMS-1566618. 
representation of $S L_{2}\left(F_{p}\right)$ for which $\rho$ is irreducible. In the case where $\rho$ is in one of the three main series of irreducible representations of $\Gamma$, a description of $\underline{\rho}$ was given by Humphreys in [H1], $[\mathrm{H} 2$.

0.4. For general $G$ let $\mathfrak{U}$ be the set of unipotent representations of $\Gamma$ (up to isomorphism). A study of the map $\rho \mapsto \rho$ in the case where $\rho$ is one of the irreducible representations of $\Gamma$ attached in $[\overline{\mathrm{DL}}, 1.9]$ to a generic character of a "maximal torus" of $\Gamma$ appears in Jantzen's paper [J1]; a study of the map $\rho \mapsto \rho$ in the case where $\rho \in \mathfrak{U}$ appears in Jantzen's paper [J2]. (The notion of unipotent representation of $\Gamma$ is defined in [DL, 7.8].)

0.5. In unpublished notes written in 1978, the author gave a conjectural description (on the level of dimensions only) of $\rho$ as an explicit linear combination of Weyl modules (see 1.1) in the case where $\rho \in \mathfrak{U}$ and $G$ has type $B_{2}, G_{2}, A_{3}, A_{4}$; for types $A_{1}, A_{2}$ this was known earlier, see 0.2 , 0.3 , Later the author found that this description has been proved to be correct when $G$ has type $B_{2}$ by Jantzen J]3 or type $G_{2}$ by Mertens $\mathrm{M}$. (A copy of $[\mathrm{M}$ ] was provided to the author by J. Humphreys.) Recently the author understood that the conjectural description in 1978 can be partly explained by a surprising (conjectural) general pattern which will be described in this paper. Namely, there should exist a family of objects $M_{w} \in \mathcal{R}_{p}^{+} \Gamma$ indexed by the "near involutions" (see 1.2) $w$ in $W$ such that for any $\rho \in \mathfrak{U}, \rho$ is an explicit linear combination of $M_{w}$ with $w$ near involutions in the twosided cell determined by $\rho$; the coefficients are natural numbers whose definition involves among other things the character table of the $J$-ring associated to the Weyl group.

\section{Recollections}

1.1. Let $B$ be a Borel subgroup of $G$ defined over $F_{p}$; let $T$ be a maximal torus of $B$ defined and split over $F_{p}$. Let $X$ be the group of characters $T \rightarrow \mathbf{k}^{*}$ with group operation written as addition. For any $\lambda \in X$ there is (up to isomorphism) at most one irreducible rational $G$-module $\mathbf{L}(\lambda)$ (over $\mathbf{k}$ ) such that $T$ acts on some $B$-stable line in $\mathbf{L}(\lambda)$ through the character $\lambda$; this is uniquely defined up to isomorphism. Let $X^{+}$be the set of all $\lambda \in X$ for which $\mathbf{L}(\lambda)$ is defined. There is a unique $\mathbf{Z}$-basis $\left\{\varpi_{i} ; i \in I\right\}$ of $X$ such that $X^{+}=\sum_{i \in I} \mathbf{N} \varpi_{i}$. For $I^{\prime} \subset I$ we set $\lambda_{I^{\prime}}=\sum_{i \in I^{\prime}} \varpi_{i} \in$ $X^{+}$.

For $\lambda \in X^{+}$let $\mathbf{V}(\lambda)$ be a rational $G$-module (over $\mathbf{k}$ ) whose character (an element of the group ring $\mathbf{Z}[X])$ is the same as that of the characteristic 0 analogue of $\mathbf{L}(\lambda)$; it is given by the Weyl character formula. Note that $\mathbf{V}(\lambda)$ is well defined up to rearrangement of its composition factors. Let $X_{p}^{+}$be the set of all $\lambda \in X^{+}$ of the form $\sum_{i \in I} n_{i} \varpi_{i}$ with $0 \leq n_{i} \leq p-1$ for all $i$. For $\lambda \in X_{p}^{+}$we denote by $V(\lambda) \in \mathcal{R}_{p}^{+} \Gamma$ and $L(\lambda) \in \mathcal{R}_{p}^{+} \Gamma$ the restriction of $\mathbf{V}(\lambda)$ and $\mathbf{L}(\lambda)$ to $\Gamma=G\left(F_{p}\right)$.

1.2. Let $W \subset \operatorname{Aut}(X)$ be the Weyl group of $G$. For any $i \in I$ there is a unique element $s_{i} \in W$ such that $s_{i} \neq 1$ and $s_{i}\left(\varpi_{j}\right)=\varpi_{j}$ for any $j \in I-\{i\}$. Recall that $W$ is a Coxeter group on the generators $\left\{s_{i} ; i \in I\right\}$. Let $w \mapsto l(w)$ be the length function of this Coxeter group. Let $w_{0}$ be the longest element of $W$; let $\nu$ be its length. For any $w \in W$ let $\mathcal{L}(w)=\left\{i \in I ; l\left(s_{i} w\right)<l(w)\right\}$.

Let $u^{1 / 2}$ be an indeterminate and let $H$ be the free $\mathbf{Q}\left[u^{1 / 2}, u^{-1 / 2}\right]$-module with basis $\left\{T_{w} ; w \in W\right\}$ and with an algebra structure as in [L3, 3.3]. Let $\hat{W}$ be the set 
of all irreducible $W$-module $E$ over $\mathbf{Q}$ (up to isomorphism). For $E \in \hat{W}$ let $E(u)$ be an $H$-module (free as a $\mathbf{Q}\left[u^{1 / 2}, u^{-1 / 2}\right]$-module) associated to $E$ as in [L2, 1.1]. There is a well defined integer $a_{E} \geq 0$ such that for $w \in W$ we have

$$
\operatorname{tr}\left(u^{-l(w) / 2} T_{w}, E(u)\right)=(-1)^{l(w)} c_{w, E} u^{-a_{E} / 2} \bmod u^{\left(-a_{E}+1\right) / 2} \mathbf{Z}\left[u^{1 / 2}\right]
$$

where $c_{w, E} \in \mathbf{Z}$ for all $w$ and $c_{w, E} \neq 0$ for some $w \in W$. (One can interpret $c_{w, E}$ in terms of the character of the irreducible representation associated to $E$ of the $J$-ring of $W$ at the basis element of the $J$-ring corresponding to $w$, see [L4, 3.5(b)].) For $w \in W$ we set $\alpha_{w}=\sum_{E \in W} c_{w, E} E$, a virtual representation of $W$. Let $\mathcal{J}$ be the set of "near involutions" of $W$ that is the set of all $w \in W$ such that $w, w^{-1}$ are in the same left cell of $W$. (If $W$ is of classical type, $\mathcal{J}$ is exactly the set of involutions in $W$.) According to [L4, 3.5] for $w \in W$ we have

$w \in \mathcal{J}$ if and only if $\alpha_{w} \neq 0$.

For $w \in W$ let $R_{w} \in \mathcal{R} \Gamma$ be as in [DL, 1.5]. By [L3, 6.17], for $w \in W$ there is a well defined object $R_{\alpha_{w}} \in \mathcal{R}^{+} \Gamma$ such that

$$
\sharp(W) R_{\alpha_{w}}=\sum_{E \in \hat{W}, y \in W} \operatorname{tr}(y, E) c_{w, E} R_{y}
$$

in $\mathcal{R} \Gamma$. Note that $R_{\alpha_{w}}$ is zero unless $w \in \mathcal{J}$.

An irreducible representation $\rho$ of $\Gamma$ (over $\mathbf{C}$ ) is in $\mathfrak{U}$ if and only if the multiplicity $\left(\rho: R_{\alpha_{w}}\right)$ is nonzero for some $w \in \mathcal{J}$.

1.3. In the examples below (types $A_{1}, A_{2}, B_{2}, G_{2}, A_{3}$ ) we write $I=\{1,2, \ldots\}$ where the notation is such that

(type $\left.A_{1}\right)$ if $\lambda=(a-1) \varpi_{1}$ with $a \geq 1$ then $\operatorname{dim} \mathbf{V}(\lambda)=a$;

(type $\left.A_{2}\right)$ if $\lambda=(a-1) \varpi_{1}+(b-1) \varpi_{2}$ with $a \geq 1, b \geq 1$ then $\operatorname{dim} \mathbf{V}(\lambda)=$ $a b(a+b) / 2$;

(type $\left.B_{2}\right)$ if $\lambda=(a-1) \varpi_{1}+(b-1) \varpi_{2}$ with $a \geq 1, b \geq 1$ then $\operatorname{dim} \mathbf{V}(\lambda)=$ $a b(a+b)(a+2 b) / 6$;

(type $\left.G_{2}\right)$ if $\lambda=(a-1) \varpi_{1}+(b-1) \varpi_{2}$ with $a \geq 1, b \geq 1$ then $\operatorname{dim} \mathbf{V}(\lambda)=$ $a b(a+b)(a+2 b)(a+3 b)(2 a+3 b) / 120$;

(type $\left.A_{3}\right)$ if $\lambda=(a-1) \varpi_{1}+(b-1) \varpi_{2}+(c-1) \varpi_{3}$ with $a \geq 1, b \geq 1, c \geq 1$ then $\operatorname{dim} \mathbf{V}(\lambda)=a b c(a+b)(b+c)(a+b+c) / 12$.

For a sequence $i_{1}, i_{2}, \ldots$ in $I$ we often write $w=i_{1} i_{2} \ldots$ instead of $w=s_{i_{1}} s_{i_{2}} \ldots$; we write $\emptyset$ instead of $w$ where $w$ is the unit element of $W$. We now describe the elements $R_{\alpha_{w}}$ in several examples. For $\rho \in \mathfrak{U}$ we write $d(\rho)=\operatorname{dim} \rho$. and

Type $A_{1}, I=\{1\}$. We have $\mathcal{J}=\{\emptyset, 1\}, \mathfrak{U}=\{1, S\}$ where $d(1)=1, d(S)=p$

$$
R_{\alpha_{\emptyset}}=1, R_{\alpha_{1}}=S \text {. }
$$

Type $A_{2}, I=\{1,2\}$. We have $\mathcal{J}=\{\emptyset, 1,2,121\}, \mathfrak{U}=\{1, r, S\}$ where $d(1)=$ $1, d(r)=p^{2}+p, d(S)=p^{3}$ and

$$
R_{\alpha_{\emptyset}}=1, R_{\alpha_{1}}=R_{\alpha_{2}}=r, R_{\alpha_{121}}=S .
$$

Type $B_{2}, I=\{1,2\}$. We have $\mathcal{J}=\{\emptyset, 1,2,121,212,1212\}, \mathfrak{U}=\left\{1, r, e_{1}, e_{2}, \theta, S\right\}$ where

$$
\begin{aligned}
& d(1)=1, d(r)=p(p+1)^{2} / 2, d\left(e_{1}\right)=d\left(e_{2}\right)=p\left(p^{2}+1\right) / 2, d(\theta)=p(p-1)^{2} / 2, \\
& d(S)=p^{4}, \\
& R_{\alpha_{\emptyset}}=1, R_{\alpha_{1}}=r+e_{1}, R_{\alpha_{2}}=r+e_{2}, R_{\alpha_{121}}=\theta+e_{2}, R_{\alpha_{212}}=\theta+e_{1}, R_{\alpha_{1212}}=S .
\end{aligned}
$$


Type $G_{2}, I=\{1,2\}$. We have $\mathcal{J}=\{\emptyset, 1,2,121,212,12121,21212,121212\}$, $\mathfrak{U}=\left\{1, r, r^{\prime}, e_{1}, e_{2}, e^{\prime}, f, g, h, S\right\}$ where

$$
\begin{gathered}
d(1)=1, d(r)=p(p+1)^{2}\left(p^{2}+p+1\right) / 6, d\left(r^{\prime}\right)=p(p+1)^{2}\left(p^{2}-p+1\right) / 2, \\
d\left(e_{1}\right)=d\left(e_{2}\right)=p\left(p^{4}+p^{2}+1\right) / 3, d\left(e^{\prime}\right)=p(p-1)^{2}\left(p^{2}-p+1\right) / 6, \\
d(f)=p(p-1)^{2}\left(p^{2}+p+1\right) / 2, d(g)=d(h)=p\left(p^{2}-1\right)^{2} / 3, d(S)=p^{6}, \\
R_{\alpha_{\emptyset}}=1, R_{\alpha_{1}}=r+r^{\prime}+e_{1}, R_{\alpha_{2}}=r+r^{\prime}+e_{2}, \\
R_{\alpha_{121}}=r^{\prime}+e_{2}+f+g+h, R_{\alpha_{212}}=r^{\prime}+e_{1}+f+g+h, R_{\alpha_{12121}}=e_{1}+e^{\prime}+f, \\
R_{\alpha_{21212}}=e_{2}+e^{\prime}+f, R_{\alpha_{121212}}=S .
\end{gathered}
$$

Type $A_{3}, I=\{1,2,3\}$. We have $\mathcal{J}=\{\emptyset, 1,2,3,13,121,232,2132,13231,121321\}$, $\mathfrak{U}=\left\{1, r, r^{\prime}, r^{\prime \prime}, S\right\}$ where

$$
\begin{gathered}
d(1)=1, d(r): p^{3}+p^{2}+p, d\left(r^{\prime}\right): p^{4}+p^{2}, d\left(r^{\prime \prime}\right)=p^{5}+p^{4}+p^{3}, d(S): p^{6}, \\
R_{\alpha_{\emptyset}}=1, R_{\alpha_{1}}=R_{\alpha_{2}}=R_{\alpha_{3}}=r, R_{\alpha_{13}}=R_{\alpha_{2132}}=r^{\prime} \\
R_{\alpha_{121}}=R_{\alpha_{13231}}=R_{\alpha_{232}}=r^{\prime \prime}, R_{\alpha_{121321}}=S .
\end{gathered}
$$

\section{The elements $M_{w} \in \mathcal{R}_{p}^{+} \Gamma$ FOR $w \in \mathcal{J}$}

2.1. In each of the examples in 1.3 and for any $w \in \mathcal{J}$ we define a virtual representation $M_{w} \in \mathcal{R}_{p} \Gamma$ as a certain integer combination of objects $V(\lambda)$. If $I=\{1,2, \ldots, s\}$ we write $V_{n_{1}, n_{2}, \ldots, n_{s}}$ instead of $V(\lambda)$ where $\lambda=n_{1} \varpi_{1}+n_{2} \varpi_{2}+\cdots+n_{s} \varpi_{s}$. We set $\delta(w)=\operatorname{dim}\left(M_{w}\right)$.

Type $A_{1}: M_{\emptyset}=V_{0}, M_{1}=V_{p-1} ; \delta(\emptyset)=1, \delta(1)=p$.

Type $A_{2}: M_{\emptyset}=V_{0,0}, M_{1}=V_{p-1,0}, M_{2}=V_{0, p-1}, M_{121}=V_{p-1, p-1}$;

$\delta(\emptyset)=1, \delta(1)=\delta(2)=p(p+1) / 2, d(121)=p^{3}$.

Type $B_{2}: M_{\emptyset}=V_{0,0}, M_{1}=V_{p-1,0}, M_{2}=V_{0, p-1}, M_{121}=V_{p-3,0}, M_{212}=V_{0, p-2}$, $M_{1212}=V_{p-1, p-1}$

$d(\emptyset)=1$,

$\delta(1)=p(p+1)(p+2) / 6$

$\delta(2)=p(p+1)(2 p+1) / 6$,

$\delta(121)=p(p-1)(p-2) / 6$,

$\delta(212)=p(p-1)(2 p-1) / 6$,

$\delta(1212)=p^{4}$.

Type $G_{2}: M_{\emptyset}=V_{0,0}, M_{1}=V_{p-1,0}, M_{2}=V_{0, p-1}, M_{121}=V_{p-4,1}, M_{212}=V_{1, p-2}$, $M_{12121}=V_{p-4,0}, M_{21212}=V_{0, p-2}, M_{121212}=V_{p-1, p-1}$;

$\delta(\emptyset)=1$,

$\delta(1)=p(p+1)(p+2)(p+3)(2 p+3) / 120$,

$\delta(2)=p(p+1)(2 p+1)(3 p+1)(3 p+2) / 120$,

$\delta(121)=p(p-1)(p+1)(p-3)(p+3) / 30$,

$\delta(212)=p(p-1)(p+1)(3 p-1)(3 p+1) / 30$,

$\delta(12121)=p(p-1)(p-2)(p-3)(2 p-3) / 120$,

$\delta(21212)=p(p-1)(2 p-1)(3 p-1)(3 p-2) / 120$,

$\delta(121212)=p^{6}$.

Type $A_{3}$ :

$M_{\emptyset}=V_{0,0,0}$,

$M_{1}=V_{p-1,0,0}$,

$M_{2}=V_{0, p-1,0}-V_{0, p-3,0}$, 


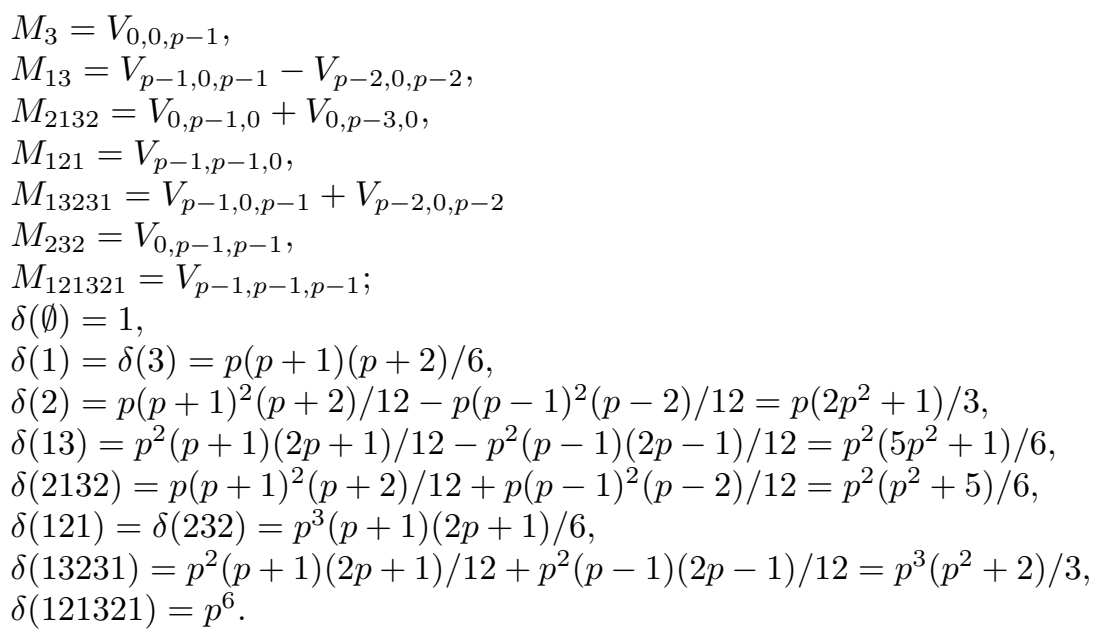

Note that in each of the cases above we have $M_{w} \in \mathcal{R}_{p}^{+} \Gamma$. (This is obvious except for type $A_{3}$ and $w=2$ or $w=13$ where it can be verified directly.)

2.2. For any $\rho \in \mathfrak{U}$ we write $\rho$ (with $\rho \in \mathfrak{U}$ ) as an $\mathbf{N}$-linear combination of $M_{w}$ (in $\left.\mathcal{R}_{p} \Gamma\right)$ in each case in 1.3 .

Type $A_{1}: \underline{1}=M_{\emptyset}, \underline{S}=M_{1}$

Type $A_{2}: \underline{1}=M_{\emptyset}, \underline{r}=M_{1}+M_{2}, \underline{S}=M_{121}$.

Type $B_{2}: \underline{1}=M_{\emptyset}, \underline{r}=M_{1}+M_{2}, \underline{e}_{1}=M_{1}+M_{212}, \underline{e}_{2}=M_{121}+M_{2}, \underline{\theta}=$ $M_{121}+M_{212}, \underline{S}=M_{1212}$.

Type $G_{2}: \underline{1}=M_{\emptyset}, \underline{r}=M_{1}+M_{2}, \underline{r}^{\prime}=M_{1}+M_{121}+M_{2}+M_{212}, \underline{e}_{1}=M_{1}+$ $M_{12121}+M_{212}, \underline{e}_{2}=M_{121}+M_{2}+M_{21212}, \underline{e}^{\prime}=M_{12121}+M_{21212}, f=M_{121}+$ $M_{12121}+M_{212}+M_{21212}, \underline{g}=\underline{h}=M_{121}+M_{212}, \underline{S}=M_{121212}$.

Type $A_{3}: \underline{1}=M_{\emptyset}, \underline{r}=\bar{M}_{1}+M_{2}+M_{3}, \underline{r}^{\prime}=M_{13}+M_{2132}, \underline{r}^{\prime \prime}=M_{121}+M_{13231}+$ $M_{232}, \underline{S}=M_{121321}$.

We return to a general $G$. We state the following

Conjecture 2.3. There exist nonzero objects $M_{w} \in \mathcal{R}_{p}^{+} \Gamma,(w \in \mathcal{J})$ such that for any $\rho \in \mathfrak{U}$ we have

(a) $\underline{\rho}=\sum_{w \in \mathcal{J}}\left(\rho: R_{\alpha_{w}}\right) M_{w}$.

Moreover, we can assume that the following properties hold.

(i) For any $w \in \mathcal{J}, M_{w}$ is a $\mathbf{Z}$-linear combination of $V_{\lambda}$ with $\lambda$ very close to $(p-1) \lambda_{\mathcal{L}(w)}($ see 1.1)

(ii) We have $\operatorname{dim}\left(M_{w}\right)=\pi_{w}(p)$ where $\pi_{w}(t) \in \mathbf{Q}[t]$ ( $t$ an indeterminate) is independent of $p$. There exists an involution $w \leftrightarrow \tilde{w}$ of $\mathcal{J}$ such that $t^{\nu} \pi_{w}(1 / t)=$ $\pm \pi_{\tilde{w}}(t)$ and $\mathcal{L}(\tilde{w})=I-\mathcal{L}(w)$ for all $w \in \mathcal{J}$

(iii) For $w \in \mathcal{J}$ we write $\pi_{w}(t) \in t^{c(w)} \mathbf{Q}[t], \pi_{w}(t) \notin t^{c(w)-1} \mathbf{Q}[t]$ where $c(w) \in \mathbf{N}$ is well defined. Then $c(w)$ depends only on the two-sided cell of $W$ containing $w$; it is the value of the a-function (see [L4, 3.1]) of $W$ on that two-sided cell.

A similar statement can be made when $F_{p}$ is replaced by the finite field $F_{p^{n}}$ with $p^{n}$ elements for some $n \geq 2$.

The conjecture does not say what the $M_{w}$ are explicitly.

2.4. By the results in 2.1, 2.2 the conjecture holds for types $A_{1}, A_{2}, B_{2}, G_{2}, A_{3}$. For these types, the involution $w \leftrightarrow \tilde{w}$ in 2.3 (ii) is given as follows: 
Type $A_{1}: \emptyset \leftrightarrow 1$;

Type $A_{2}: \emptyset \leftrightarrow 121,1 \leftrightarrow 2$;

Type $B_{2}: \emptyset \leftrightarrow 1212,1 \leftrightarrow 2,121 \leftrightarrow 212$;

Type $G_{2}: \emptyset \leftrightarrow 121212,1 \leftrightarrow 2,121 \leftrightarrow 212 ; 12121 \leftrightarrow 21212$;

Type $A_{3}: \emptyset \leftrightarrow 121321,1 \leftrightarrow 232,2 \mapsto 13231,3 \mapsto 121,13 \leftrightarrow 2132$.

Similar evidence exists for type $A_{4}$.

2.5. Here is the simplest nontrivial example of objects $M_{w}$ in 2.3. Assume that $V$ is a three dimensional $F_{p}$-vector space and $\Gamma=S L(V)$. Let $Z_{1}$ be the set of lines in $V$. Let $Z_{2}$ be the set of planes in $V$. Let $\mathcal{F}_{1}$ be the vector space of functions $Z_{1} \rightarrow \mathbf{k}$ with sum of values equal to 0 . Let $\mathcal{F}_{2}$ be the vector space of functions $Z_{2} \rightarrow \mathbf{k}$ with sum of values equal to 0 . Note that $\mathcal{F}_{1}, \mathcal{F}_{2}$ are naturally $\Gamma$-modules; they both represent $\rho$ where $\rho \in \mathfrak{U}$ has dimension $p^{2}+p$. Define $\tau: \mathcal{F}_{1} \rightarrow \mathcal{F}_{2}$ by $\left(\tau(f)(P)=\sum_{L \in Z_{1} ; L \subset P} f(L)\right.$ where $f \in \mathcal{F}_{1}, P \in Z_{2}$. Define $\tau^{\prime}: \mathcal{F}_{2} \rightarrow \mathcal{F}_{1}$ by $\left(\tau^{\prime}\left(f^{\prime}\right)(L)=\sum_{P \in Z_{2} ; L \subset P} f^{\prime}(P)\right.$ where $f^{\prime} \in \mathcal{F}_{2}, L \in Z_{1}$. Note that $\tau, \tau^{\prime}$ are well defined $\Gamma$-linear maps. Let $M$ be the kernel of $\tau$ (it is also the image of $\tau^{\prime}$ ). Let $M^{\prime}$ be the kernel of $\tau^{\prime}$ (it is also the image of $\tau$ ). Then $M, M^{\prime}$ are the objects $M_{w}$ attached to $\rho$ in 2.3 .

2.6. If in the sum 2.3(a) we replace each $M_{w}$ by the basis element $t_{w}$ of the $J$-ring of $W$ (see [L4, 3.5]), the resulting element of the $J$-ring is contained in the centre of that ring.

2.7. A statement similar to 2.3(a) can be made for any, not necessarily unipotent, irreducible representation $\rho$ of $\Gamma$. (We replace the $J$-ring of $W$ and the left cells of $W$ by the analogous ring $H_{\mathcal{O}}^{\infty}$ and the left cells considered in [L5, 1.9] in terms of an extended Weyl group.) We illustrate this in an example.

Let $\mathcal{O}$ be an orbit for the obvious $W$-action on $X /(p-1) X$ such that the stabilizer in $W$ of any element of $\mathcal{O}$ is trivial. For any $\zeta \in \mathcal{O}$ there is a unique element $\tilde{\zeta} \in X_{p}^{+}$whose image under $X \rightarrow X /(p-1) X$ equals $\zeta$. Let $\zeta_{0} \in \mathcal{O}$. Let $\tilde{\zeta}_{0}^{\prime}$ be the composition $T \stackrel{\tilde{\xi}_{0}}{\longrightarrow} \mathbf{k}^{*} \stackrel{\phi^{\prime}}{\longrightarrow} \mathbf{C}^{*}$ where $\phi^{\prime}$ is the homomorphism such that $\phi\left(\phi^{\prime}(x)\right)=x$ for any $x \in \mathbf{k}^{*}(\phi$ as in 0.1$)$. We can restrict $\tilde{\zeta}_{0}$ to $T\left(F_{p}\right)$ and we regard this restriction as a homomorphism $B\left(F_{p}\right) \rightarrow \mathbf{C}^{*}$ trivial on the Sylow $p$ subgroup of $B\left(F_{p}\right)$. This last homomorphism can be induced to a representation $\rho$ of $\Gamma$ over $\mathbf{C}$ which is in fact irreducible and depends only only on $\mathcal{O}$, not on $\zeta_{0}$. From the results of [CL, $\rho$ has each of $L(\tilde{\zeta}),(\zeta \in \mathcal{O})$ as a composition factor (but it may also have other composition factors). We expect that

(a) $\underline{\rho}=\sum_{\zeta \in \mathcal{O}} M_{\zeta}$

where $M_{\zeta} \in \mathcal{R}_{p}^{+} \Gamma$ is a $\mathbf{Z}$-linear combination of various $V(\lambda)$ with $\lambda \in X_{p}^{+}$very close to $\tilde{\zeta}$.

In the case where $G=S L_{3}(\mathbf{k})$ such a statement can be deduced from [CL] (in this case we have $M_{\zeta}=V(\tilde{\zeta})$ for each $\left.\zeta \in \mathcal{O}\right)$; in the case where $G=S p_{4}(\mathbf{k})$, a statement like (a) can be deduced from [J3].

\section{REFERENCES}

[BN] R. Brauer and C. Nesbitt, On the modular characters of groups, Ann. of Math. (2) 42 (1941), 556-590, DOI 10.2307/1968918. MR 4042

[CL] R. W. Carter and G. Lusztig, Modular representations of finite groups of Lie type, Proc. London Math. Soc. (3) 32 (1976), no. 2, 347-384, DOI 10.1112/plms/s3-32.2.347. MR396731 
[DL] P. Deligne and G. Lusztig, Representations of reductive groups over finite fields, Ann. of Math. (2) 103 (1976), no. 1, 103-161, DOI 10.2307/1971021. MR393266

[H1] James E. Humphreys, Ordinary and modular representations of Chevalley groups, Lecture Notes in Mathematics, Vol. 528, Springer-Verlag, Berlin-New York, 1976. MR0453884

[H2] J. E. Humphreys, Ordinary and modular characters of $\mathrm{SL}(3, p)$, J. Algebra 72 (1981), no. 1, 8-16, DOI 10.1016/0021-8693(81)90309-4. MR634614

[J1] Jens Carsten Jantzen, Zur Reduktion modulo p der Charaktere von Deligne und Lusztig (German), J. Algebra 70 (1981), no. 2, 452-474, DOI 10.1016/0021-8693(81)90229-5. MR623819

[J2] Jens Carsten Jantzen, Zur Reduktion modulo p unipotenter Charaktere endlicher ChevalleyGruppen (German), Math. Z. 181 (1982), no. 1, 97-128, DOI 10.1007/BF01214985. MR671718

[J3] J. C. Jantzen, Representations of Chevalley groups in their own characteristic, The Arcata Conference on Representations of Finite Groups (Arcata, Calif., 1986), Proc. Sympos. Pure Math., vol. 47, Amer. Math. Soc., Providence, RI, 1987, pp. 127-146, DOI 10.1016/s00224049(99)00142-5. MR933356

[L1] G. Lusztig, On the discrete representations of the general linear groups over a finite field, Bull. Amer. Math. Soc. 79 (1973), 550-554, DOI 10.1090/S0002-9904-1973-13198-2. MR.315010

[L2] George Lusztig, Unipotent characters of the symplectic and odd orthogonal groups over a finite field, Invent. Math. 64 (1981), no. 2, 263-296, DOI 10.1007/BF01389170. MR629472

[L3] George Lusztig, Characters of reductive groups over a finite field, Annals of Mathematics Studies, vol. 107, Princeton University Press, Princeton, NJ, 1984. MR742472

[L4] G. Lusztig, Leading coefficients of character values of Hecke algebras, The Arcata Conference on Representations of Finite Groups (Arcata, Calif., 1986), Proc. Sympos. Pure Math., vol. 47, Amer. Math. Soc., Providence, RI, 1987, pp. 235-262, DOI 10.1007/bf01389157. MR 933415

[L5] G. Lusztig, Conjugacy classes in reductive groups and two-sided cells, Bull. Inst. Math. Acad. Sin. (N.S.) 14 (2019), no. 3, 265-293, DOI 10.21915/bimas.2019301. MR4033057

[M] D. Mertens, Zur Darstellungstheorie der endlicher Chevalley-Gruppen von typ G , Diplo- $^{2}$ marbeit (1985), Universität Bonn.

Department of Mathematics, Massachusetts Institute of Technology, Cambridge, MassachusetTs 02139

Email address: gyuri@mit.edu 